\title{
Evaluation of Two Fundamental Positive-Sequence Detectors for Highly Distorted and Unbalanced Systems
}

\author{
Helder Carneiro, Bruno Exposto, João L. Afonso \\ Centro Algoritmi - Departamento de Electrónica Industrial \\ Univ. Minho - Campus de Azurém 4800-058 - Guimarães, Portugal \\ E-mail: hcarneiro@dei.uminho.pt; bexposto@dei.uminho.pt; jla@dei.uminho.pt
}

\begin{abstract}
This paper presents an evaluation of the performance of two different Positive-Sequence Detectors. This evaluation is made by comparing a PLL (Phase-Locked- Loop), combined with Lagrange Multipliers Method, with an Adaptive Filter. Both algorithms aim to detect the positive sequence at the fundamental frequency on a given three-phase system voltages or currents. The purpose of this evaluation is to assess the better Positive-Sequence Detector for custom power devices, such as Active Power Filters. The comparative analysis was based on the evaluation of a series of steady state performance parameters (phase and amplitude errors, THD and unbalance) and on the response time. The tests were made to study the behavior of both approaches when working with highly distorted and unbalanced signals. This work was carried out using the computer simulation tool PSCAD/EMTDC.
\end{abstract}

Keywords; Active Power Filters; Phase-Locked- Loop; Adaptive Band-Pass Filter

\section{INTRODUCTION}

The electrical power systems of nowadays suffers from different power quality issues with several causes, but all leading to financial losses due to failure in fabrication processes or information systems. These problems are harmonics, inter-harmonics, flicker, notches, sags and swells; amongst others.

In order to mitigate these power quality issues, research has been done to develop equipment that improves power quality on electrical power systems. In 1976, Gyugyi and Strycula introduced the first power quality devices, denominated Active Filters [1]. Further investigation resulted in the introduction of several devices used to compensate most of the power quality problems: Dynamic Voltage Restorers (DVR) [2], Unified Power Quality Conditioners (UPQC) [3], Uninterruptable Power Supply (UPS) [4][5], Active Power Filters (APF)[6][7][8], and others. Most of these conditioners have their control strategies based on the real time detection of the positive sequence at the fundamental frequency of the system voltages or currents. Thus it is very important to use detectors that accurately calculate these components. This is also important for other applications related with power quality. For instance, it can be used in measurement devices [9], PFC converters [10], and, are employed in renewable energy interface, by syncing renewable energy sources with the electrical power grid [10][11]. The wide scope of fields that require detection of real time detection of the positive sequence at the fundamental frequency of the system voltages or currents makes this paper relevant.

The major objective of this paper is to analyze two different fundamental positive-sequence detectors (PLL and Adaptive Band-Pass Filter) when dealing with highly distorted and unbalanced signals. This paper first describes the PLL (Phase-Locked- Loop) Control Circuit basic operation, together with a control algorithm based on the Lagrange Multiplier Method; and the Adaptive Band-Pass Filter. Then, the simulation environment and performance parameters used to analyze both positive sequence detectors are described. Further on, the simulation results are presented, and the behavior of both approaches is analyzed and compared. Finally, conclusions are taken.

\section{PLL CONTROL CIRCUIT}

The PLL Control Circuit tracks the positive sequence at the fundamental frequency, even in conditions of highly distorted and unbalanced three phase signals (voltages or currents) [12]. Fig. 1 illustrates the PLL circuit design. This synchronizing circuit determines, in real time, the frequency and phase angle of the measured signals fundamental positive-sequence component, which, in this case, corresponds to the per-unit phase currents $i_{S a}, i_{S b}$, and $i_{S c}$. The algorithm is based on the instantaneous active three-phase power $\left(p_{3 \phi}\right)$, given on (1), which, in a system where the sum of the phase voltages is zero $\left(v_{a}+v_{b}+v_{c}=0\right)$, can be rewritten by (2):

$$
\begin{aligned}
& p_{3 \phi}=v_{a} \cdot i_{S a}+v_{b} \cdot i_{S b}+v_{c} \cdot i_{S c}, \\
& p_{3 \phi}=v_{a} \cdot i_{S a b}+v_{c} \cdot i_{S c b},
\end{aligned}
$$

where: $i_{S a b}=i_{S a}-i_{S b}$ and $i_{S c b}=i_{S c}-i_{S b}$.

The voltage feedback signals of Fig. $1, v_{a}(\omega t)=\sin (\omega t)$ and $v_{c}(\omega t)=\sin (\omega t+2 \pi / 3)$, are generated by the PLL circuit using the time integral of $\omega$. It should be noticed that these signals have unitary amplitude and that $v_{c}(\omega t)$ leads $v_{a}(\omega t)$ by $120^{\circ}$. Thus, they represent a feedback from a positive sequence component at an angular frequency $\omega$. The variable $p_{3 \phi}$ is the input of the PI-controller. 


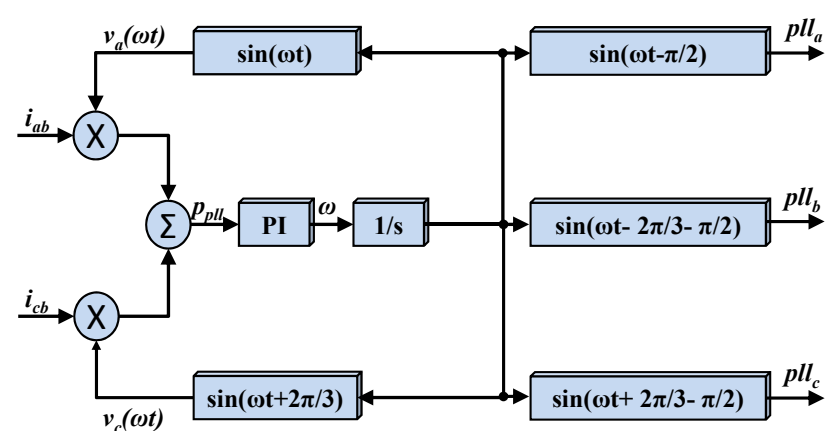

Fig. 1 - PLL Control Circuit.

The PLL circuit can reach a stable point of operation only if the average part of $p_{3 \phi}\left(\bar{p}_{3 \phi}\right)$ has zero value, and if its low frequency oscillating part, $\tilde{p}_{3 \phi}\left(\tilde{p}_{3 \phi}=p_{3 \phi}-\bar{p}_{3 \phi}\right)$, has been minimized. In terms of phasors, $\bar{p}_{3 \phi}$ can be calculated according to (3):

$$
\bar{p}_{3 \phi}=3 \cdot \dot{V}_{+1} \cdot \dot{I}_{+1} \cdot \cos \phi .
$$

When this circuit achieves stability, the PI controller output $(\omega)$ corresponds to the fundamental angular frequency, and the feedback signal $v a(\omega t)$ leads the fundamental positive sequence component of the measured phase current $i_{a s}$ by $90^{\circ}$ [13][14].

\section{A. Lagrange Multiplier Method}

Since the PLL Control Circuit produces only unitary output signals, it is used a control algorithm based on the Lagrange Multiplier Method to retrieve the correct amplitude of the measured signals (current signals, for the case described in this paper). This method calculates, in real time, the magnitude of the fundamental positive-sequence components. The calculations inputs are the system currents, $i_{S a}, i_{S b}, i_{S c}$, and the PLL output signals, $p l l_{a}, p l l_{b}, p l l_{c}$.

It is calculated a fictitious conductance, $G$ [15], according to the Lagrange Multiplier Method:

$$
G=\frac{p l l_{a} \cdot i_{S a}+p l l_{b} \cdot i_{S b}+p l l_{c} \cdot i_{S c}}{\left(p l l_{a}\right)^{2}+\left(p l l_{b}\right)^{2}+\left(p l l_{c}\right)^{2}} .
$$

This parameter can be compared with the instantaneous equivalent conductance of the FDB power theory [16]. In order to extract only the average component from $G$, a sliding average filter is employed. The sliding average filter is constituted by sliding average that determines the mean value of input signals. By doing this from the initial signal are removed any high frequencies. This average signal, denominated in Fig. 2 as $G_{b a r}$, comprehends the fundamental positive-sequence magnitude of the system currents. Thus, the direct product between $G_{b a r}$ and the PLL output signals, $p l l_{a}, p l l_{b}, p l l_{c}$, results in signals that correspond to the system currents fundamental positive sequence components, as expected.

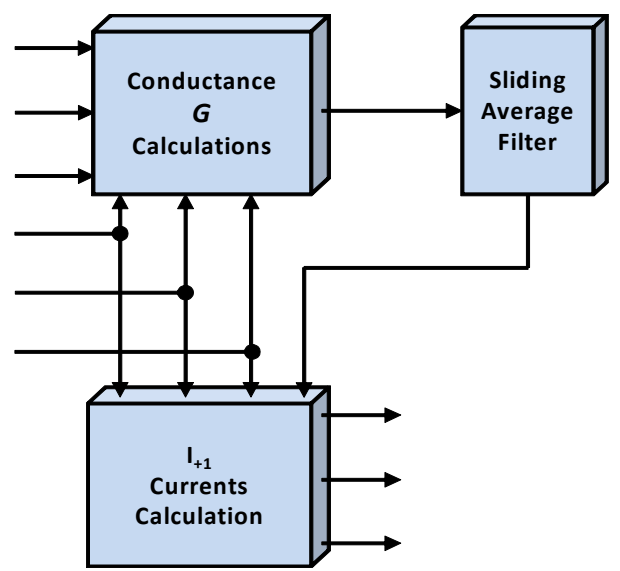

Fig. 2 - Lagrange Multiplier Method.

The resulted signals are denominated $i_{a w}, i_{b w}, i_{c w}$ :

$$
\left\{\begin{array}{l}
i_{a w}=G_{b a r} \cdot p l l_{a} \\
i_{b w}=G_{b a r} \cdot p l l_{b} \\
i_{c w}=G_{b a r} \cdot p l l_{c}
\end{array}\right.
$$

\section{ADAPTIVE BAND PASS FILTER}

The Adaptive Band-Pass Filter can be applied to detect the positive sequence at the fundamental frequency of a given three-phase signal type (voltage or current) [17].

Fig. 3 shows the block diagram of the Adaptive Filter in the Laplace domain.

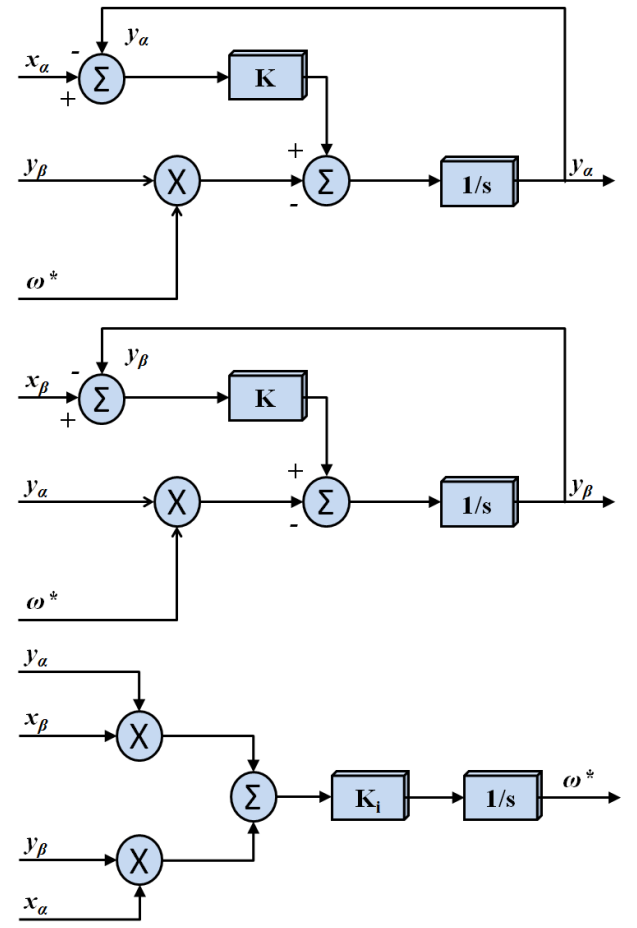

Fig. 3 - Block diagram of the Adaptive Band-Pass Filter. 
The parameters and variables of the filter are:

- $K$ - proportional gain;

- $K_{i}$ - gain applied in the calculation of the central frequency;

- $\omega-$ angular frequency of the signals;

- $x_{\alpha}$ e $x_{\beta}$ - input variables of the filter in the $\alpha-\beta$ referential;

- $y_{\alpha}$ e $y_{\beta}-$ output variables of the filter, represented in the $\alpha-\beta$ referential.

The first step consists of applying the Clarke transform to convert the three-phase signals from the $a-b-c$ referential to the $\alpha-\beta$ referential:

$$
\left[\begin{array}{l}
x_{\alpha} \\
x_{\beta}
\end{array}\right]=\sqrt{\frac{2}{3}} \cdot\left[\begin{array}{ccc}
1 & -1 / 2 & -1 / 2 \\
0 & \sqrt{3} / 2 & -\sqrt{3} / 2
\end{array}\right] \cdot\left[\begin{array}{c}
i_{a} \\
i_{b} \\
i_{c}
\end{array}\right]
$$

The input of the Adaptive Band-Pass Filter is a generic vector $X$, with amplitude $A$ and angular frequency $\omega$. The coordinates $x_{\alpha}$ and $x_{\beta}$ are given by:

$$
\left[\begin{array}{l}
x_{\alpha} \\
x_{\beta}
\end{array}\right]=A \cdot\left[\begin{array}{l}
\cos (\omega \cdot t) \\
\sin (\omega \cdot t)
\end{array}\right]
$$

The variation of the vector $X$ in the time is given by:

$$
\frac{d X}{d t}=\frac{d}{d t}\left[\begin{array}{c}
x_{\alpha} \\
x_{\beta}
\end{array}\right]=\omega \cdot\left[\begin{array}{c}
-x_{\beta} \\
x_{\alpha}
\end{array}\right] .
$$

The variation of the output signals $y_{\alpha}{ }^{\prime}$ and $y_{\beta}{ }^{\prime}$ in the time domain can be obtained from (8), together with a feedback of the output signals $y_{\alpha}$ and $y_{\beta}$. This feedback guarantees the stability of the Adaptive Band-Pass Filter.

$$
\left[\begin{array}{l}
y_{\alpha}{ }^{\prime} \\
y_{\beta}{ }^{\prime}
\end{array}\right]=K \cdot\left[\begin{array}{l}
x_{\alpha}-y_{\beta} \\
x_{\beta}-y_{\beta}
\end{array}\right]+\omega \cdot\left[\begin{array}{c}
-y_{\beta} \\
y_{\alpha}
\end{array}\right] \text {. }
$$

In order to obtain the output signals $y_{\alpha}$ and $y_{\beta}$ it is only necessary to integrate the previous expression.

$$
\left[\begin{array}{l}
y_{\alpha} \\
y_{\beta}
\end{array}\right]=\int\left[\begin{array}{l}
y_{\alpha}{ }^{\prime} \\
y_{\beta}{ }^{\prime}
\end{array}\right] \cdot d t .
$$

In a real electrical system the frequency is not always kept constant in $50 \mathrm{~Hz}$. Thus, if $\omega$ is defined as a constant value, the performance of the Adaptive Band-Pass Filter can be considerably compromised if the frequency varies from its value. Therefore, it is necessary to estimate the fundamental frequency of the input signals, which is achieved by applying (11),

$$
\omega^{*}=\int\left[\left(y_{\alpha} \cdot x_{\beta}-y_{\beta} \cdot x_{\alpha}\right) \cdot K_{i}\right] \cdot d t
$$

Where the gain $K_{i}$, determines the response time of the Adaptive Filter.

In order to convert the positive sequence of the fundamental frequency from $\alpha-\beta$ to the three-phase system $a$ $b-c$, it is applied the inverse Clarke transform, as it can be seen in (12), where the three-phase positive sequence of the fundamental component are represented as $i_{a F}-i_{b F}-i_{c F}$ :

$$
\left[\begin{array}{l}
i_{a F} \\
i_{b F} \\
i_{c F}
\end{array}\right]=\sqrt{\frac{2}{3}} \cdot\left[\begin{array}{cc}
1 & 0 \\
-1 / 2 & \sqrt{3} / 2 \\
-1 / 2 & -\sqrt{3} / 2
\end{array}\right] \cdot\left[\begin{array}{l}
y_{\alpha} \\
y_{\beta}
\end{array}\right] .
$$

\section{SIMULATION}

The simulation model was implemented to test the behavior of both fundamental positive sequence detection approaches in a number of situations. The frequency of the system voltages does not remain constant in some of the tests, and the loads were chosen to test the suitability of the filters operating in highly distorted and unbalanced conditions.

The parameters used in both approaches were calculated according to the guidelines presented in [18]. These values were not modified throughout the simulations, in order to test the capability of the filters to adapt to different working conditions as they occur.

The model simulates an electrical circuit that contains a three-phase rectifier bridge with a parallel RC load, and three different passive single-phase loads connected to each phase. The RC load of the rectifier consists of a $10 \Omega$ resistor and a $4 \mathrm{mF}$ capacitor. It consumes $29.7 \mathrm{~kW}$ and its currents Total Harmonic Distortion (THD) is approximately $56 \%$. The single-phase passive load connected to phase $a$ is a series RC load $\left(R_{a}=10 \Omega\right.$ and $\left.C_{L}=1 \mathrm{mF}\right)$ which consumes $4.8 \mathrm{~kW}$ and has a power factor of 0.95 . Phase $b$ has a purely resistive load $\left(R_{b}=14 \Omega\right)$ which consumes $3.8 \mathrm{~kW}$. Phase $c$ has a RL load $\left(R_{c}=10 \Omega\right.$ and $\left.\mathrm{L}_{\mathrm{L}}=50 \mathrm{mH}\right)$ with a power factor of 0.54 and consumes a power of $1.5 \mathrm{~kW}$. These loads can be seen in Fig. 4. The power supply consists of a three-phase four wire system, $230 \mathrm{~V}$ (phase to neutral), $50.0 \mathrm{~Hz}$.

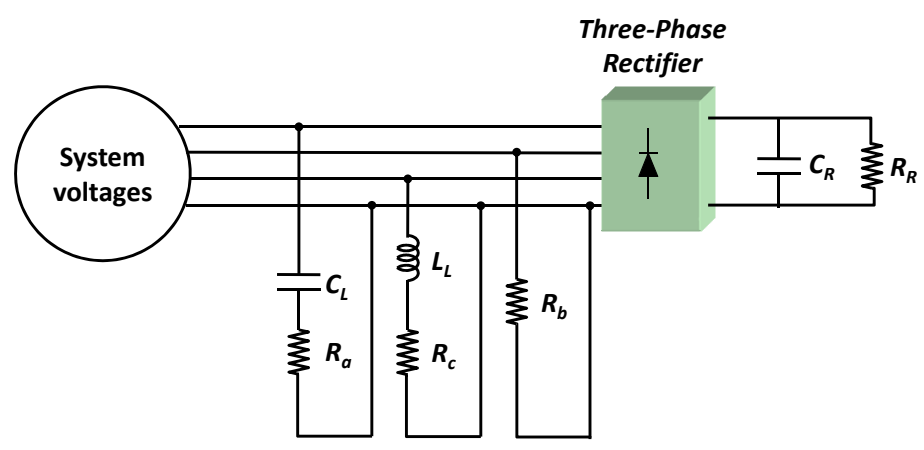

Fig. 4 - Modeled electrical circuit and loads. 
The parameters used to compare the performance of both filters outputs were:

- $\quad$ Phase angle error $(P h e)$ - This value, measured after the system reaches steady state, gives the error in degrees between the fundamental component of the positive sequence calculated by a Fourier transform and the output of the filters.

- Amplitude Error $(A e)$ - It is the difference between the peak value of the fundamental component of the positive sequence calculated by a Fourier transform and the output of the filters. This value is a percentage and, like the previous variable, it is calculated after the system reaches steady state.

- Total Harmonic Distortion of the output values of the filters $(T H D)$ - It is the ratio between the RMS value of the total harmonic content and the RMS value of the fundamental, given in percentage.

- Unbalance $(U)$ - This value is a measure of the unbalance of three-phase signals in steady state. According to the IEEE 1159-1995 standard [19], imbalance (unbalance) can be estimated as the maximum deviation from the average of the threephase voltages or currents, divided by the average of the three-phase voltages or currents, expressed in percent.

- Response time $\left(t_{r}\right)$ - It is given by the difference between the instant that the output reaches $98 \%$ of its nominal value and the instant that the input is changed. This value is expressed in milliseconds.

\section{A. Simulation Procedures}

The simulation model and the following results were obtained using the PSCAD/EMTDC software. During the simulations the following procedures, here described, were used. Initially, only the three-phase non-linear load was connected. Once the system reached steady state, both the PLL and the Adaptive Filter were connected, at the same time (at $t=100 \mathrm{~ms}$ ). All the parameters were measured once the system reached steady state (except $t_{r}$ which was measured between the time that the filters were connected until they reach steady state). The next step consisted in turning on the single-phase linear loads introducing unbalance to the system. It was possible to determine the flexibility of different approaches as unbalance was introduced and the current wave forms changed. These loads were connected one at a time and there was an interval of 1 second between each connection. Once again all the parameters described above were measured. When the simulation reached $t=4 \mathrm{~s}$ the threephase load was disconnected.

\section{B. Simulation Results}

During the simulations, the first stage consisted in observing the behavior of the PLL and Adaptive Filter with a high value of THD (55\%), but with balanced currents. Fig. 5 shows the system currents, the output signals of both approaches in steady state, and the positive sequence component at the fundamental frequency calculated through the method of the Symmetrical Components. This method was used as a quality reference in steady state for the performance of the PLL and Adaptive Filter.

In the intermediate stages each of the single-phase loads was connected bringing unbalance to the load currents (and at the same time changing the power factor). Fig. 6 shows the system currents, fundamental positive sequence currents and outputs of both approaches when two of the single-phase loads are turned on. The unbalance of the system currents was $22 \%$.

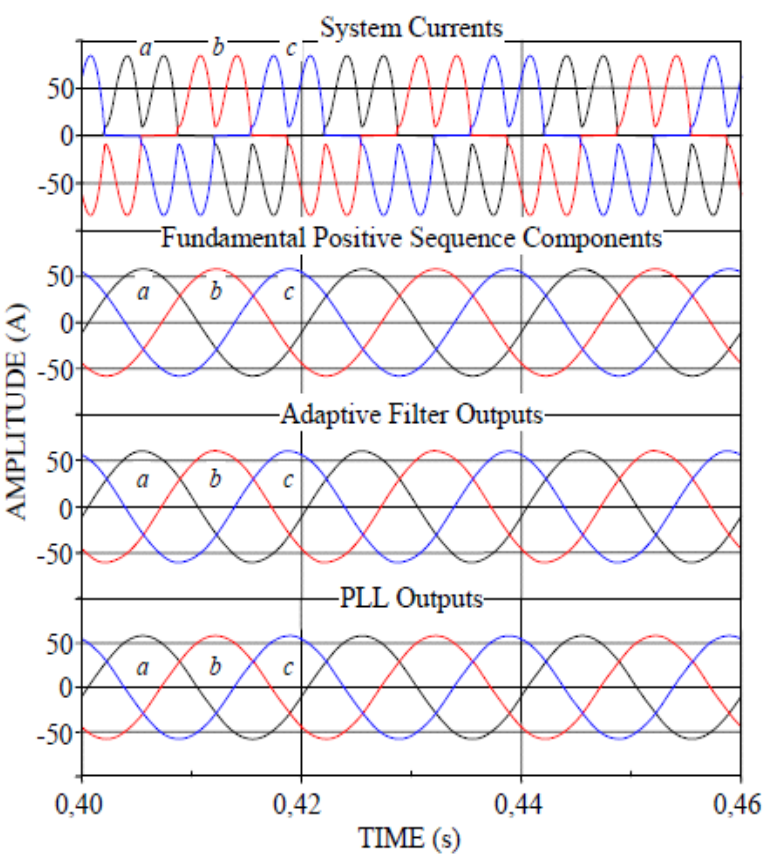

Fig. 5 - Currents with three-phase non-linear load.

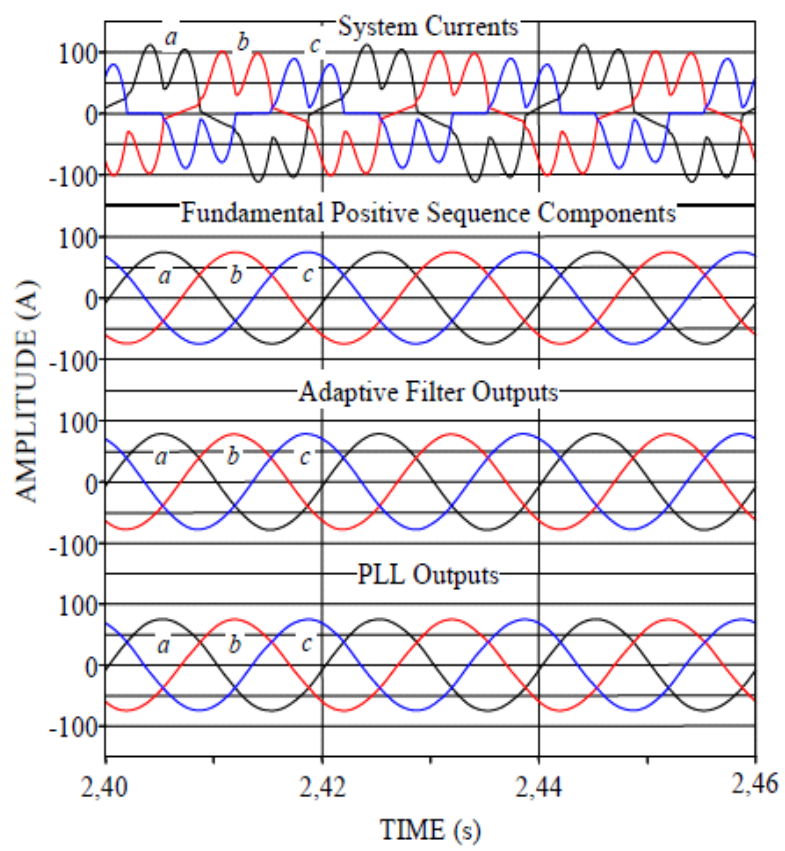

Fig. 6 - Currents with unbalanced non-linear loads. 


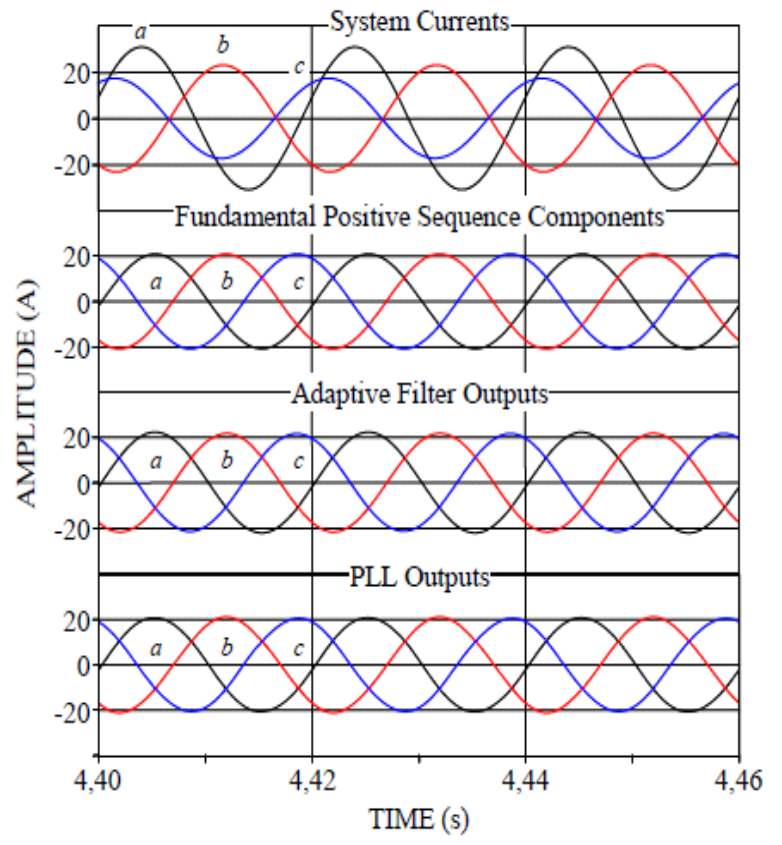

Fig.7 - Currents with unbalanced linear loads.

Finally, Fig. 7 also shows the same variables when the three-linear loads were connected, and the three-phase rectifier was disconnected. In this case, although there was practically no harmonic distortion, the unbalance of the system currents was $32 \%$.

Table I show the different performance parameters when only the three-phase load was connected. Analyzing these results it is possible to conclude that $P h_{e}$ is inferior to $1 \mathrm{deg}$ in both cases, although the error of the Adaptive Filter is over $50 \%$ inferior to the error of the PLL. Concerning $t r$, the PLL has a slightly better performance, as the steady state is reached in 5 and a half cycles against the 6 cycles needed by the Adaptive Filter. $A_{e}$ is much smaller at the output of the PLL, only $0.1 \%$, against the $4 \%$ of the Adaptive Filter. Concerning the parameters $U$ and $T H D$, both approaches have a very similar behavior.

Table II presents the performance parameters when the single-phase load of phase $a$ is turned on. It can be seen that some parameters of the PLL worsen relatively to the adaptive filter: the THD of the PLL becomes the double of the Adaptive Filter; $U$ turns into almost four times superior; and $P h_{e}$ also increases $(1.1 \mathrm{deg}$ against $0.3 \mathrm{deg}$ of the Adaptive Filter). On the other hand, $A_{e}$ remains much smaller to the PLL $(0.7 \%$ to the PLL and $4.1 \%$ to the Adaptive Filter); and the same applies to $t_{r}$ (1 cycle to the PLL and 3 to the Adaptive Filter).

Table III shows the results obtained when the linear loads of phases $a$ and $b$ are connected to the system. Once again $P h_{e}, U$ and THD are worse in the PLL, and conversely, $t_{r}$ and $A_{e}$ are worse in the Adaptive Filter.
TABLE I

RESULTS WITH THE NON-LINEAR LOAD CONNECTED

\begin{tabular}{|l|c|c|}
\cline { 2 - 3 } \multicolumn{1}{c|}{} & Adaptive Filter & PLL \\
\hline $\boldsymbol{t}_{\boldsymbol{r}}(\boldsymbol{m s})$ & $120^{*}$ & $110^{*}$ \\
\hline $\boldsymbol{A}_{\boldsymbol{e}}(\boldsymbol{\%})$ & 4.0 & 0.1 \\
\hline $\boldsymbol{P h}_{\boldsymbol{e}}(\boldsymbol{d e g})$ & 0.3 & 0.8 \\
\hline $\boldsymbol{U}(\boldsymbol{\%})$ & 0.1 & 0.1 \\
\hline $\boldsymbol{T H D}(\%)$ & 0.7 & 0.8 \\
\hline
\end{tabular}

TABLE II

RESULTS WITH THE NEW LOAD CONNECTED IN PHASE A

\begin{tabular}{|c|c|c|}
\cline { 2 - 3 } \multicolumn{1}{c|}{} & Adaptive Filter & PLL \\
\hline $\boldsymbol{t}_{\boldsymbol{r}}(\boldsymbol{m s})$ & $60^{*}$ & $20^{*}$ \\
\hline $\boldsymbol{A}_{\boldsymbol{e}}(\boldsymbol{\%})$ & 4.1 & 0.7 \\
\hline $\boldsymbol{P} \boldsymbol{h}_{\boldsymbol{e}}(\boldsymbol{d e g})$ & 0.3 & 1.1 \\
\hline $\boldsymbol{U}(\%)$ & 0.3 & 1.1 \\
\hline $\boldsymbol{T H D}(\%)$ & 0.6 & 1.2 \\
\hline & * Approximate value. \\
\hline
\end{tabular}

TABLE III

RESULTS WITH TWO SINGLE-PHASE LOADS CONNECTED

\begin{tabular}{|l|c|c|}
\cline { 2 - 3 } \multicolumn{1}{c|}{} & Adaptive Filter & PLL \\
\hline $\boldsymbol{t}_{\boldsymbol{r}}(\boldsymbol{m s})$ & $60^{*}$ & $20^{*}$ \\
\hline $\boldsymbol{A}_{\boldsymbol{e}}(\boldsymbol{\%})$ & 4.1 & 0.7 \\
\hline $\boldsymbol{P h}(\boldsymbol{d e g})$ & 0.3 & 1.1 \\
\hline $\boldsymbol{U}(\%)$ & 0.3 & 1.1 \\
\hline $\boldsymbol{T H D}(\%)$ & 0.6 & 1.2 \\
\hline
\end{tabular}

Table IV compiles the data obtained when only the linear single-phase loads are connected. There is no harmonic distortion, but there is a high level of unbalance (in amplitude and phase). The performance in terms of this parameter $(U)$ was this time better for the PLL, only $1.9 \%$, against $2.9 \%$ for higher for the Adaptive Filter, but the phase angle was correct in just 2 cycles (only the amplitude needed approximately $190 \mathrm{~ms}$ to reach steady state). The THD in the PLL was higher than in the Adaptive Filter (with only $0.01 \%$ ) and the $P h e$ was $1.1 \mathrm{deg}$ for both filters. The parameter $A_{e}$ remained similar to the other tests.

TABLE IV

RESULTS OBTAINED WITH LINEAR LOADS

\begin{tabular}{|l|c|c|}
\cline { 2 - 3 } \multicolumn{1}{c|}{} & Adaptive Filter & PLL \\
\hline $\boldsymbol{t}_{\boldsymbol{r}}(\boldsymbol{m s})$ & $190^{*}$ & $36^{*}$ \\
\hline $\boldsymbol{A}_{\boldsymbol{e}}(\boldsymbol{\%})$ & 4.2 & 0.7 \\
\hline $\boldsymbol{P h} \boldsymbol{h}_{\boldsymbol{e}}(\mathbf{d e g})$ & 0.7 & 0.7 \\
\hline $\boldsymbol{U}(\%)$ & 1.3 & 1.0 \\
\hline $\boldsymbol{T H D}(\%)$ & 0.01 & 1.3 \\
\hline \multicolumn{3}{|c|}{ "Approximate value. } \\
\hline
\end{tabular}


One last test consisted in changing the system frequency while the simulation was running. The initial value was 49.75 $\mathrm{Hz}$, being shifted to $50.25 \mathrm{~Hz}$ (limit values for the EN 50190 standard [20]) when $t=2.5 \mathrm{~s}$. Both filters kept working, managing to lock on the correct fundamental frequency and positive sequence phase, in less than one cycle. The performance of both filters did not show any noticeable degradation when the frequency changed.

\section{CONCLUSIONS}

From the analysis of the simulation results, it was possible to conclude that, both the PLL and the Adaptive Filter are valid solutions for the implementation of fundamental positive sequence detectors.

If the purpose is to use a fundamental positive sequence detector that stabilizes rapidly, and where the amplitude error is an important factor, then the PLL is better suited to accomplish the task. However, it should be noted that the Adaptive Filter takes longer to stabilize only in terms of amplitude, taking normally less than two cycles to stabilize in terms of phase angle when loads are inserted or withdrawn. On the other hand, if the preferred characteristics are a very low THD and a low phase angle error, then the Adaptive Filter presents a better behavior and should be considered. Regarding unbalance, the Adaptive Filter has generally a better performance; however this is not always true, as it was seen in the test where only the linear loads were operating The phase error was little higher with PLL in the tests, except in the case with linear loads, in which was equal. The amplitude error is always higher with Adaptive Filter that with PLL.

\section{ACKNOWLEDGMENTS}

This work is financed by FEDER Funds, through the Operational Programme for Competitiveness Factors COMPETE, and by National Funds through FCT Foundation for Science and Technology, under the project PTDC/EEA-EEL/104569/2008.

\section{REFERENCES}

[1] L. Gyugyi and E.C. Strycula, “Active ac Power Filters," IEEE Ind App Soc Annual Meeting, vol. 19-C, 1976, pp. 529-535.

[2] C. Fitzer, M. Barnes, and P. Green, "Voltage Sag Detection Technique for a Dynamic Voltage Restorer," IEEE Transactions on Industry Applications, vol. 40, Jan. 2004, pp. 203-212.

[3] M.J.V. Aredes, M.; Neto, J.A.M.; Ferreira, J.C.C.; Monteiro, L.F.C.; Fernandes, R.M.; Siqueira, "A Simplified Control Strategy for a Unified Power Quality Conditioner Prototype," Power Electronics Specialists Conference, 2005. PESC '05. IEEE 36th, 2005, pp. 2592 2597
[4] J. Chen and C. Chu, "Combination Voltage-Controlled and CurrentControlled PWM Inverters for UPS Parallel Operation," IEEE Transactions on Power Electronics, vol. 10, 1995, pp. 547-558.

[5] R.M.S. Filho, P.F. Seixas, P.C. Cortizo, B. Torres, and A.F. Souza "Comparison of three single-phase PLL algorithms for UPS applications," IEEE Transactions on Industrial Electronics, vol. 55, 2008, pp. 2923-2932.

[6] F.D. Freijedo, J. Doval-Gandoy, Ó. Lopez, P. Fernandez-Comesana, and C. Martinez-Penalver, "A Signal-Processing Adaptive Algorithm for Selective Current Harmonic Cancellation in Active Power Filters," IEEE Transactions on Industrial Electronics, vol. 56, Aug. 2009, pp. 2829-2840.

[7] F.D. Freijedo, J. Doval-Gandoy, Ó. Lopez, and E. Acha, “A Generic Open-Loop Algorithm for Three-Phase Grid Voltage/Current Synchronization With Particular Reference to Phase, Frequency, and Amplitude Estimation," IEEE Transactions on Power Electronics, vol. 24, Jan. 2009, pp. 94-107.

[8] E. Lavopa, P. Zanchetta, M. Sumner, and F. Cupertino, "Real-Time Estimation of Fundamental Frequency and Harmonics for Active Shunt Power Filters in Aircraft Electrical Systems," IEEE Transactions on Industrial Electronics, vol. 56, Aug. 2009, pp. 2875-2884.

[9] A. Cataliotti, V. Cosentino, and S. Nuccio, “A Phase-Locked Loop for the Synchronization of Power Quality Instruments in the Presence of Stationary and Transient Disturbances," IEEE Transactions on Instrumentation and Measurement, vol. 56, 2007, pp. 2232-2239.

[10] L. Huber, B.T. Irving, and M.M. Jovanovi $\underset{\iota}{ }$, "Review and Stability Analysis of PLL-Based Interleaving Control of DCM/CCM Boundary Boost PFC Converters," IEEE Transactions on Power Electronics, vol. 24, Aug. 2009, pp. 1992-1999.

[11] J. Choi, Y. Kim, and H. Kim, "Digital PLL control for Single-Phase Photovoltaic System," IEE Proceedings Electric Power Applications, vol. 153, 2006, pp. 40-46.

[12] M. Cacciato, A. Consoli, R. Attanasio, and F. Gennaro, "SoftSwitching Converter With HF Transformer for Grid-Connected Photovoltaic Systems," IEEE Transactions on Industrial Electronics, vol. 57, May. 2010, pp. 1678-1686.

[13] M. Aredes, L.F.C. Monteiro, and J. Mourente, "Control Strategies for Series and Shunt Active Filters," Power Tech Conference Proceedings, 2003 IEEE Bologna, vol.2, 2003, p. 6pp.

[14] H. Carneiro, L.F.C. Monteiro, and J.L. Afonso, "Comparisons between synchronizing circuits to control algorithms for single-phase active converters," 2009 35th Annual Conference of IEEE Industrial Electronics, Nov. 2009, pp. 3229-3234.

[15] M. Aredes and L. F. C. Monteiro, "A control strategy for shunt active filter," in Harmonics and Quality of Power, 2002. 10th International Conference on, 2002, vol. 2, pp. 472-477.

[16] H.K.M. Paredes, F.P. Marafão, and L.C.P. Silva, "Current Decompositions - Part I : Three-Phase Three-Wire Systems," PowerTech, 2009 IEEE, 2009, pp. 1-8.

[17] M.A.P. Esteve, “Aplicación de Nuevas técnicas de Controlo para el Desarrollo de Reguladores Activos de Potencia” 2002.

[18] D.R. Costa, L.G.B. Rolim, and M. Aredes, "Analysis and Software Implementation of a Robust Synchronizing Circuit - PLL Circuit," Industrial Electronics, 2003. ISIE '03. IEEE International Symposium on , vol.1, 2003, pp. 292-297.

[19] IEEE Std 1159 ${ }^{\mathrm{TM}}-2009$, IEEE Recommended Practice for Monitoring Electric Power Quality, 2009.

[20] "EN50160-Caractéristiques de la Tension Fournie par les Réseaux Publics de Distribution," 2000. 